Erratum: Analytical Sciences, 2017, Vol. 33, No. 12, p. 1441

\title{
A Biotin-streptavidin-enhanced Carbon Nanotube Amplification Strategy for an Ultrasensitive Immunodetection of Polybrominated Diphenyl Ethers
}

\author{
Xiaohan Zhang and Huisheng ZhUANG ${ }^{\dagger}$ \\ School of Environment Science and Technology, Shanghai Jiao Tong University, Minhang District, \\ Shanghai 200240, P. R. China
}

On page 1441 , the affiliation of the authors,

School of Environment Science and Technology, Shanghai Jiao Tong University, Minhang District, Shanghai 200240, P. R. China

should read

School of Environmental Science and Engineering, Shanghai Jiao Tong University, Minhang District, Shanghai 200240, P. R. China. 